\title{
Malaria Control in Complex Humanitarian Emergencies
}

\author{
John R. MacArthur, Holly A. Williams \& Peter B. Bloland
}

\begin{abstract}
War, famine, civil conflict, and political persecution displacing large populations often leads to severe disruptions in health services, disease control programs, food distribution systems, and loss of shelter. When the dimensions of the crisis overwhelm the local and international communities' ability to respond quickly and effectively, significant morbidity and mortality result in what is termed a complex humanitarian emergency. The public health consequences have been most severe in underdeveloped nations where most deaths are caused by communicable diseases, which include malaria. This paper describes and analyses the factors that contribute to malaria morbidity and mortality and proposes effective measures to combat them.
\end{abstract}

\section{Résumé}

Les conflits armés, les famines, les guerres civiles, les persécutions politiques déplaçant de grandes portions de la population provoquent souvent de graves perturbations dans les services de santé,

Dr. MacArthur is currently an Epidemic Intelligence Officer with the Malaria Branch of the Centers for Disease Control and Prevention. His interests include evaluation of antimalarial drug resistance, malaria drug policy, and malaria control in complex humanitarian emergencies.

Dr. Williams is a Medical Anthropologist with the Malaria Branch of the Centers for Disease Control and Prevention. Currently, her research interests include malaria control in complex emergencies, drug resistance, drug policy, and sociobehavioral issues related to malaria control.

Dr. Bloland is a medical epidemiologist with the Malaria Branch of the Centers for Disease Control and Prevention. His current research interest is in the role of artemisinin antimalarial drugs in preventing the development of drug resistance. les programmes de contrôles sanitaires, les structures de distributions alimentaires, et entraînent fréquemment la perte dugite ou del'abris. Quandl'ampleurde la crise submerge les capacités locales et internationales à y répondre promptement et efficacement, le résultat de ceque l'on appelle une urgence humanitaire complexe est un accroissement significatif de la condition maladive et de la mortalité des populations en cause. Les conséquences en termes de santépublique sont particulièrement graves dans les nations sous-développées, où un plus grand nombre de pertes de vie sont dues à des maladies transmissibles, incluant notamment la malaria. Cetarticle décrit et analyse la série de facteurs contribuant à la condition maladive et à la mortalité liés à la malaria, et propose des mesures effectives pour combattre ces facteurs.

\section{Introduction}

In 1998, there were an estimated 30 million refugees or internally displaced persons in need of protection and assistance worldwide. ${ }^{1}$ War, famine, civil conflicts, and political persecution continue to result in mass displacement of populations. These events often contribute to severe disruptions in health services, disease control programs, food distribution systems, and loss of shelter. Large populations are commonly displaced from underdeveloped areas into new sites with scarce resources. When the emergency reaches the dimensions where it overwhelms both the local and international communities' ability to respond quickly, significant morbidity and mortality occur due to severe public health problems. This situation is known as a complex humanitarian emergency.

Mortality rates are used to divide complex humanitarian emergencies into two phases: emergency and post- emergency. ${ }^{2}$ The emergency phase typically lasts from 1 to 6 months and is characterized by outbreaks of communicable diseases and crude mortality rates above one death per 10,000 persons per day. ${ }^{3}$ The second stage of the crisis is the post-emergency or maintenance phase. During this period, the health profile is similar to that of the host country. The displaced population, however, continues to be at risk for the same communicable diseases that are present in the surrounding areas. ${ }^{4}$

The intent of this paper is to review basic concepts of malaria and malaria control for non-malariologists and to discuss how these concepts can be applied in the context of complex humanitarian emergencies. Successful implementation of malaria controlearly in an emergency situation can minimize the impact of this potentially severe disease among the displaced population.

\section{Malaria}

\section{Routes of Transmission}

Malaria infection in humans is caused by one or more of four species of intracellular parasites. Plasmodium falciparum, P. vivax, P. ovale, and P. malariae all cause disease although each clinical course is different. P. vivax is the most common species that infects humans worldwide, while P. falciparum is the most serious form leading to severe or fatal malaria. These parasites are most commonly transmitted by the infective bite of a female Anopheles species mosquito but can alsobe transmitted through blood transfusions and transplacentally from mother to fetus. 


\section{Epidemiology}

Malaria occurs in more than 90 countries and $36 \%$ of the world's population live in areas with risk of malaria transmission. An additional $29 \%$ reside in areas once malaria free but now experiencing resurgent transmission, and $7 \%$ live in areas where the disease has never been under meaningful control. Each year there are an estimated 300-500 million clinical cases of malaria resulting in approximately 1.5-2.7 million deaths. ${ }^{5}$

Malaria transmission occurs in Central and South America, the Caribbean island of Hispaniola (Haiti and Dominican Republic), the Middle East, Central Asia, the Indian subcontinent, southern China, Southeast Asia, Korea, and Oceania. Most of malaria deaths, however, occur in children under 5 years of age living in sub-Saharan Africa.

Chloroquine-resistant P. falciparum was first recognized in Thailand and South America in the late 1950s. Since then, it has spread and intensified to the point where only parts of Central America, Hispaniola, and the Middle East are free of chloroquine (CQ) resistance. Drug resistance is not limited to $\mathrm{CQ}$ alone. Resistance is emerging to sulfadoxine/pyrimethamine (SP) in parts of eastern sub-Saharan Africa, Southeast Asia, and the Amazon region of South America. The border regions of Thailand have reported mefloquine resistance as well (figure). ${ }^{6}$ Chloroquineresistant $P$. vivax has been reported in Southeast Asia and Oceania and, in some foci, can be very common. ${ }^{7,8}$

\section{Clinical Features}

Infections with malaria present with a variety of signs and symptoms. The severity of the disease depends on the infecting species and the immune status of the patient. Those persons who have experienced repeated exposure to the parasite develop partial immunity and thus can better tolerate malaria infections. The risk of severe illness and death is much lower in these persons. Common symptomsinnonimmuneper- sons infected with malaria include fever, chills, sweats, myalgias, arthralgias, headache, diarrhea, and other nonspecific complaints. Signs may include splenomegaly and common laboratory abnormalities are anemia and thrombocytopenia. Patients with partial immunity have even morenonspecific symptoms, often presenting similarly to a mild viral infection.

Uncomplicated malaria can rapidly progress into severe disease and death. Severe cases are almost uniformly due to $P$. falciparum. These patients may present with neurological complications such as sleepiness, altered mental status, convulsions, and/or coma. Renal failure, pulmonary edema, hemoglobinuria, and shock can also develop. The case-fatality rate of initially non-severe falciparum malaria has been estimated at less than $5 \%$. Once the illness progresses to severe malaria, the case fatality rate is $15 \%$ $20 \%{ }^{9}$

\section{Diagnosis}

Early diagnosis and prompt effective treatment are paramount for the prevention of mortality from malaria. In disease-endemic areas, or in situations with patients arriving from these areas, all cases of febrile illness must be suspected as malaria. Information about the patient load in the clinic, time required to perform the test, costs involved, level of training and supervision necessary, and available equipment and electricity is necessary before deciding which diagnostic tool is best to use.

Presumptive diagnosis is commonly used in many parts of the world, especially in sub-Saharan Africa where it is simple, inexpensive, and rapid. However, diagnosis based on signs and symptoms is not reliable due to thenonspecific nature of clinical malaria. This poor predictive value, even in areas of intense transmission, will lead to overdiagnosis and, thus, unnecessary use of antimalarial medications. This can have adverse effects on the patient and possibly lead to increased antimalarial drug resistance. Over-diagnosis of malaria also causes the health care worker to miss other possibly important causes of the fever.

The current leading diagnostic method is microscopic examination of Giemsa-stained, thick-and-thin peripheral blood smears. Advantages of microscopy include high sensitivity and specificity among properly trained and supervised technicians. Microscopy also offers the ability to identify the infecting species and quantify the level of parasitemia. Although this is the "gold standard," setting up microscopy in the emergency phase of a complex humanitarian emergency may be difficult. In relief settings in Africa, where malaria transmission can be intense, microscopy-based diagnosis may be difficult if the number of febrile patients overwhelms the ability of the laboratory to examine the slides rapidly. Programs using laboratory diagnosis must ensure appropriate quality control of the tests. Although this may be difficult in some settings, there are quality control reports from refugee camps showing high sensitivity and specificity. ${ }^{10}$

Rapid diagnostic tests offer state-ofthe-art technology for malaria control efforts. These tests require no special equipment or electricity, can be performed after minimal training, do not need cold storage, and offer high sensitivity and specificity. They are principally used to detect P. falciparum, although new tests also detect nonfalciparum species. The major disadvantage of these tests is a high per-test cost (currently US $\$ 1$ - $\$ 2$ per test). Another disadvantage, especially in areas where drug resistance is a major problem, is the persistence of positivity after treatment. The test can remain positive up to 14 or more days after treatment, ${ }^{11}$ prohibiting their use for monitoring parasite response after treatment.

Anemia is a frequent complication of malaria and contributes significantly to the overall morbidity and mortality associated with malaria. ${ }^{12}$ Therefore, evaluation of anemia should be a component of a control program. Factors such as poor food rations, helminth 
infections, recent diarrheal illnesses, hereditary hemoglobinopathies, and low socioeconomic status also contribute to decreased hemoglobin levels. Accurate clinical diagnosis based on pallor depends on the experience of the practitioner, but is a method that often yields poor results. ${ }^{13}$ Hematocrit centrifuges are extremely sensitive. However, they require electricity for optimal performance. Hemoglobinometers are also very sensitive, but are expensive to buy and operate. One option that is affordable, accurate, and easy to use consists of special filter paper and a color scale. ${ }^{14}$

\section{Case Management}

Rapid and effective case management is the cornerstone of malaria control efforts. Falciparum malaria can progress to fatal disease within 48 hours, and therefore an effective first line therapy is essential. Optimally, treatment varies depending on the infecting species, local drug-resistance patterns, and severity of disease. If diagnostic facilities capable of determining species are not available, all presumptive cases must be treated as P. falciparum since this is the most lethal form of malaria.

Drug resistance patterns should be understood before the establishment of antimalarialdrug policies. Generally, if the area is known to have P. falciparum parasites sensitive to $\mathrm{CQ}$ (Central America, Hispaniola, Egypt, and limited regions of the Middle East) then it can be selected as the drug of choice.

In areas where multiple drug resistance exists, the preferred option for the treatment of uncomplicated $P$. falciparum is the combination of an artemisinin derivative (e.g., artesunate) for 3 days with another antimalarial drug such as mefloquine. Studies investigating the combination of artesunate and SP are currently under way. This combination may offer a highly efficacious option for areas where CQ resistance is high but $S P$ remains effective. In some refugee settings in Southeast Asia, artemisinin combined with mefloquine appears to have slowed the development of drug resistance and decreased malaria transmission. ${ }^{15}$

Care of patients with severe $P$. falciparum should include the use of a fast-acting antimalarial drug, prevention and treatment of convulsions and other complications, and good nursing care. Many deaths result due to delay in treatment or inappropriate treatment. Quinine remains the treatment of choice in patients with severe malaria. Other ancillary treatments may include antipyretics, anticonvulsants, furosemide, and dextrose. ${ }^{16}$

In areas of significant malaria risk, all anemic patients should be treated with antimalarial drugs regardless of the cause of the anemia. Micronutrient supplementation with iron, folate, Vitamin A, Vitamin C, or zinc can further enhancehematologic recovery. Caution is advised in using folate supplementation in conjunction with SP as treatment failures may increase. ${ }^{17}$ Blood transfusions used to treat severe anemia can be lifesaving. However, there are risks involved with transfusions if screening for blood-borne pathogens is not reliable. Transfusions in patients with a hemoglobin level $<5 \mathrm{~g} / \mathrm{dl}$ with respiratory distress or all those with hemoglobin $<4 \mathrm{~g} / \mathrm{dl}$ have been shown to improve survival. ${ }^{12}$ Due to the high risk of acquiring HIV from infective donors, all blood must be screened before transfusion. This may be impractical in the emergency phase of the crisis and thus a referral program to the nearest hospital with transfusion capabilities should be instituted. If this is not practical, the relief organization must rapidly develop blood transfusion protocols that include HIV screening.

In areas of the world where women have low levels of immunity, malaria is a major cause of maternal mortality, abortion, stillbirths, premature birth, and low birth weight. ${ }^{18}$ In populations with high levels of immunity, malaria contributes to maternal anemia and low birth weight babies. Because of this, special attention needs to be given to provide rapid and effective curative services to pregnant women to reduce excess malaria-related mortality.

\section{Preventive Services}

Rarely does the relief agency have control over the selection of the resettlement site of a displaced population. Geographic features of the selected area may contribute to the transmission of malaria. Even with the influx of relief operations, shelter in the emergency phase is often crude, thus exposing the population to anopheline mosquitoes and possible malaria infection.

In pregnant women, weekly prophylaxis with an effective drug (e.g., mefloquine in the second and third trimesters) can prevent complications. 19,20 Intermittent therapy, the routine presumptive treatment with an effective antimalarial drug given once during the second and third trimesters, is a costeffective method to prevent complications in semi-immune women in areas of high transmission. In populations with high-HIV seropositivity, intermittent therapy needs to be given more often. ${ }^{21-23}$

Mass prophylaxis is not generally recommended for populations exposed to perennial malaria transmission. However, there may be some high risk groups, such as pregnant women, very young children, or malnourished persons who may benefit from prophylaxis. ${ }^{19,20}$

Efforts with vector control are often minimal in the early stages of the emergency. Information about local vector behaviour may be obtained from the host country malaria control staff. If this is not available and if staffing permits, early operational studies of the vector population, especially biting behavior, can be useful. Vector control takes a more prominent role in the postemergency phase when the camp becomes more stable with the appearance of houses and other structures.

Distribution of insecticide-treated bednets is one preventive measure that should be undertaken. Nets can be hung even in crude shelters and are effective in the prevention of malaria. Although untreated nets may reduce the risk of malaria transmission, those treated 
with an insecticide offer much better protection. All insecticide-treated net programs should have a component of training on how to reimpregnate the nets as the insecticide is only effective for 4 to 6 months. Community participation is essential if the program is to be successful. If the community has no prior experience with bednets, information and education on their use must accompany the distribution. Unless the displaced population understands the purpose of the nets and "buys into" the program, the nets may be sold for alternative items that the community perceives to be of greater importance or value.

Indoor residual spraying of insecticides may be an appropriate large-scale vector control measure for some settings, especially during a complex humanitarian emergency, as it does not require behavior change. For spraying to be fully effective, insecticide must be applied to all interior surfaces of all houses at regular intervals and is only effective against vectors that bite and rest indoors after a blood meal. ${ }^{24}$

When there are only a few sites responsible for mosquito breeding (e:g., pond), chemical larvicides can be used. Biological larvicides, such as certain species of fish, may be employed to control the mosquito population and also provide the community with an extra protein source. When there are many potential breeding sites, the use of larvacides is generally not cost effective.

Another vector control measure is breeding site reduction. Examples include controlling the runoff from water stations or filling holes. With the exception of Anopheles stephensi in southwest Asia, artificial containers such as pots or tubs are not suitable for anophelinebreeding. A thorough knowledge of the behavior of the local anopheline mosquito is imperative for appropriate control.

\section{Complex Humanitarian Emergencies}

\section{General}

Despite years of effort to reduce the impact of malaria on mortality rates, it continues to be a major cause of death in the tropical and subtropical developing world. Most complex emergenciessince the early 1960s have taken place in under developed countries where local resources have been insufficient to provide fast and effective relief services. ${ }^{25}$ In many of these crises malaria was endemic in both the country of origin and the country of asylum.

While the malaria burden in these countries can be great, it is exacerbated where there is a mass migration of people. Displaced persons are at increased risk of acquiring malaria for several reasons. The geography of endemic areas and the population's acquired immunity to the disease are another important aspect affecting the vulnerability to acquiring malaria. If the displaced population moves from a malaria-free area to an endemic zone, it is at increased risk for malaria infections, epidemics, severe illness, and death. When a group flees a disease-endemic region and seeks refuge in a malaria-free area, it may introduce the parasites into the host community, thus posing risks for the local inhabitants.

When large populations are forced to move, they frequently travel long distances to arrive at an area felt to be secure. During the journey, they sleep outside and are exposed to mosquito bites. Upon arrival, they often settle in uninhabited areas and near water sources. Many will bring livestock with them that may attract more mosquitoes. ${ }^{26,27}$ Initially, the group lives in open areas without adequate shelter, potable water, or sanitation. These camps are often overcrowded and have no formal health care facilities. Environmental damage caused by settling into new areas also contributes to the development of vector breeding sites.

\section{Epidemic Malaria}

Due to the aforementioned characteristics of displaced populations, the risk of epidemic malaria is very high. The initial response to an apparent epidemic of malaria is to confirm an increase in cases, determine the extent of the outbreak, and investigate the demo- graphics of those infected. Once the presence of an epidemichas been established, additional support and logistical resources should be rapidly mobilized to assist in the management. The key components of epidemic control include the provision of effective curative therapy to the affected population, prevention of further transmission, containment of the outbreak, and improvement of readiness for future epidemics. $^{24}$

Early warning surveillance systems should be designed to assist in the detection of epidemics. Monitoring the number of cases of fever and malaria on a daily or weekly basis can alert the relief team to an unusual increase in incidence. Other variables such as rainfall, temperature, and vector density may also provide useful information in predicting epidemics.

Management of an epidemic differs depending on the infecting species, size of the outbreak, and resources available to the intervention team. The team should rely on the full use of available refugee health workers to educate the public, conduct active case detection, organize testing, and dispense treatment. Ideally, only diagnostically confirmed cases of malaria are treated, but this may be unrealistic in settlements with large populations. Another option is to confirm the presence of an outbreak with available laboratory diagnostic tests and then presumptively treat all cases of fever as malaria.

The combination of an artemisinin drug for 3 days (e.g., artesunate) with a longer acting agent for 1 day (e.g., mefloquine) has been used in Southeast Asia for P. falciparum epidemics. Although combination therapy is a good theoretical choice for epidemics as it has reduced the gametocyte carriage rate among treated individuals, ${ }^{28}$ it has not been used in epidemics in areas outside Asia.

Prompt detection, early intervention with an effective antimalarial drug, and active community participation will decrease the morbidity and mortality associated with malaria epidemics. 


\section{Malaria Control - Emergency Phase}

\section{Initial Assessment}

A comprehensive approach to conducting a needs assessment of the crisis must occur. The displaced population is in dire need of water, food, shelter, sanitation, and security. Communicable diseases such as diarrhea, measles, and meningitis contribute to increases in morbidity and mortality. ${ }^{25}$ Depending on the prevalence of malaria in the area of the resettlement, a large number of emergency phase deaths may result. ${ }^{29-}$ ${ }^{32}$ Therefore, it is imperative that relief experts begin planning for malaria control from the beginning of the emergency.

The initial malaria assessment should focus on three areas: epidemiology of the disease, environmental factors contributing to its transmission, and local entomologic data that may assist in vector control activities (see table on page 10). Some of thesedatacan be collected before the relief team departs for the field.

A situation analysis must also include information on the logistics involved in caring for the health needs of the displaced population. Questions concerning access of transportation to the selected site, availability of local materials and human resources, and legalities of the importation of relief materials (e.g., medications, supplies) must be addressed. Finally, a continual review of the safety of the site should occur. Agencies need to have a written security policy with personnel following the guidelines.

\section{Health Information System}

In the emergency phase of complex humanitarian emergencies, mortality rates can exceed 60 times the normal rates. ${ }^{2}$ Establishment of a reliable, timely surveillance system for communicable diseases endemic to the preflight and resettlement area will capture these events. This system monitors cause and age-specific mortality rates and should have the sensitivity to detectepidemics, enabling rapid interventions.

The case definition of malaria depends upon the diagnostic capabilities available. Ideally, the definition would be based on a thick smearblood slide or positive results from a rapid diagnostic test. In the early phase of the emergency, however, diagnosis may rely solely upon patients having fever or a history of fever and no other obvious infection accounting for the febrile disease.

Data should be collected and analyzed on a daily basis during the

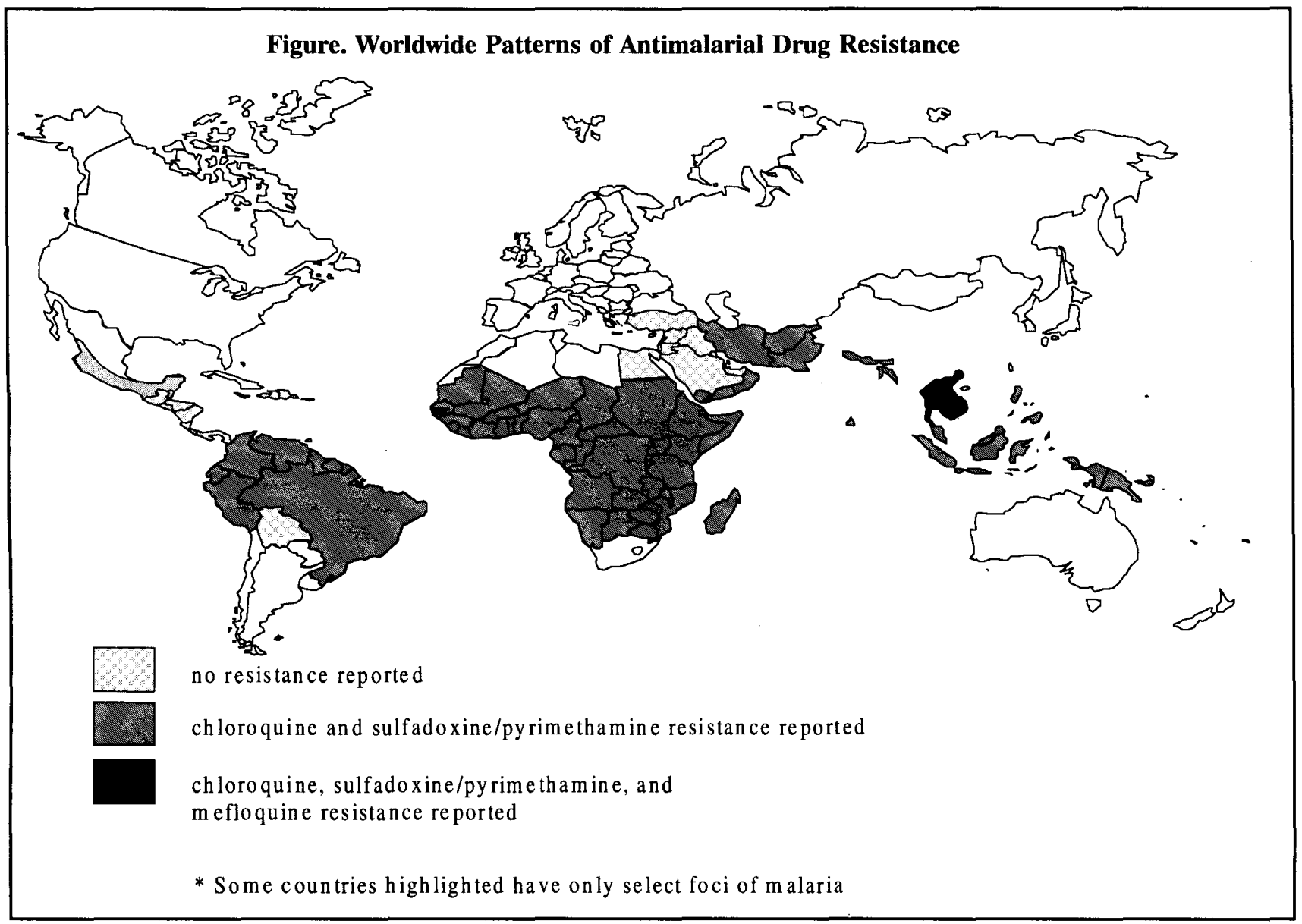


emergency phase. Graphing of crude incidence and mortality rates assists in monitoring for possible outbreaks. Data obtained from local malaria control officials can be used as baseline rates if available.

\section{Community Participation}

Outreach health programs using community health educators trained from the displaced population need to be organized early. These educators circulate among the newly arrived persons to assess the health status of the families, triage ill persons to established health centers, and provide education on prevention of the major communicable diseases prevalent. Visits to persons who arrived earlier to the camp may detect unreported illnesses and facilitate referrals to the health center.

Successful malaria control efforts include an understanding of the social behaviours of the host and displaced communities. This task may be extremely difficult to accomplish because of competing demands in meeting priority needs during the first month of operations. Attention should be placed on the various social organizations established in the camp. It is essential to understand and integrate community leaders into malaria control activities from the beginning as they may be valuable resources in supporting control activities. Also, early integration of traditional or community healers into malaria control efforts may prevent conflicts with relief agencies that promote western health care.

Information about malaria knowledge, beliefs, and practices of the community needs to be obtained Participatory or rapid appraisal methods, such as using key informants and focus groups, allow the community to share, enhance, and analyze their knowledge of life and conditions, to create a plan, and to act. ${ }^{33}$

\section{Malaria Control Post-Emergency Phase}

\section{Health Information System}

The post-emergency phase of complex emergencies is characterized by a steep decline in crude mortality rates. The focus of the health information system continues to be the collection of reliable, timely data. Training of refugee health staff in the collection and analysis of simple data should occur. These courses need to include the recognition of epidemics, and the utilization of surveillance data for monitoring, evaluation and planning of the malaria control program. If not possible during the emergency phase, case definitions of malaria should now shift to laboratoryconfirmed cases with reporting of the infecting species. Data continues to be collected, analyzed, and graphed on a daily or weekly basis, as the risk for epidemics still exists.

\section{Community Participation}

During the post-emergency phase, the displaced population settles in the new area and attempts to reconstruct the social networks that were present in their preflight communities. This is a time when people struggle with what has occurred and are in the process of redefining a sense of meaning to their lives.

Relief interventions now focus not only on curative care, but on preventive care as well. Expanded training of the community health educators selected from various groups within the displaced population continues. Relief workersneed to cooperate with the refugee team to develop culturally appropriate health education messages.

Utilization of key community members in the planning of malaria control activities allows refugees to regain a sense of control. This enhances the acceptance level of the program within the displaced community. During programmatic planning for future work, relief organizations and these community representatives need to prioritize the malaria control agenda. Decisions about further training, vector control strategies, community health education events, and choices for appropriate drug policies are made in a collective manner. Community participation is aimed at inclusion and building capacity. Both the relief organizations and the displaced people must be moving into a development mode with projected plans for community-based malaria control strategies.

\section{Other Activities}

Establishment of community health workers in the prevention and treatment of malaria and other diseases occurs in the post-emergency phase. This can be done by using medical skills already present in the community or by building capacity by training new health workers. The integration of these workers can free relief agency staff from actual patient care responsibilities and allow them to take on a supervisory role. The ultimate goal is to have the refugee health team assume the management responsibilities of the hospital and clinics.

Assessment for drug resistance must occur every 1 to 2 years to ensure that the current therapy provided is efficacious. Other operational research projects, such as community-based qualitative studies, might help in both case management and developing appropriate health education messages.

\section{Coordination}

The response to complex humanitarian emergencies by the host government and international community can be effective. However, there are often several competing organizations with similar agendas vying for a piece of the relief operation. This can be extremely problematic unless adequate coordination exists. Often the task of coordination falls to the United Nations High Commissioner for Refugees if the displaced population crosses an international border. If the population is internally displaced, the United $\mathrm{Na}$ tions Organization for Coordination of 
Humanitarian Assistance is often the lead agency.

Actual implementation of the relief efforts is usually carried out by nongovernmental organizations such as Médecins sans Frontières (medical services), OXFAM (water and sanitation), or the International Rescue Committee (education and training). Host country ministries of health or national Red Cross/Crescent societies may also be involved with implementation of relief activities. Other agencies such as the International Committee of the Red Cross may play a role as well. Efforts by the international agencies to incorporate local staff into the relief work should be made. Each organization brings certain specialties to the relief effort, however, because of the possibility of competing interests and the complexity of the emergency, coordination among agencies is paramount.

\section{Conclusion}

Malaria continues to be a major communicable disease in the tropics and subtropics, killing 1.5-2.7 million people each year. It is characteristically a disease of the poor, young, and weak. War, civil strife, mass migration of nonimmune groups into high-risk areas, breakdown of health services and malaria control programs, environmental degradation, and problems with access to the population contribute to increased morbidity and mortality.

Malaria must be recognized by the international relief community as a major cause of morbidity and mortality in the displaced population and host communities. The challenge is to implement malaria-control activities that are evidence-based, cost effective, and feasible, given the difficult nature of complex humanitarian emergencies. International relief agencies are often staffed by medical staff from developed countries with little or no experience with malaria. Therefore, by improving relief workers' understanding of malaria control concepts through adequate training before placement in the field, countless deaths due to malaria can be avoided.

\section{References}

1. USCR World Refugee Survey. 1998. Washington, DC: US Committee for Refugees, 1998.
2. Toole, M. J. and R. J. Waldman. Prevention of Excess Mortality in Refugee and Displaced Populations in Developing Countries. JAMA 1990;263(24):3296-302.

3. Bureau for Refugee Programs. Assessment Manual for Refugee Emergencies. Washington, DC: US Dept of State, 1985.

4. Burkholder, B. T. and M. J. Toole . Evolution of Complex disasters. Lancet 1995;346(8981):1012-5.

5. WHO. World Malaria Situation in 1993, part I. Wkly Epidemiol Rec 1996;71:17-22.

6. Nosten, F., F. ter Kuile, and T. Chongsuphajaisiddhi et al. Mefloquineresistant falciparum malaria on the ThaiBurmese border [see comments]. Lancet 1991;337(8750):1140-3.

7. Fryauff, D. J., S. Tuti, and A. Mardi et al. Chloroquine-Resistant Plasmodium Vivax in Transmigration Settlements of West Kalimantan, Indonesia. Am J Trop Med Hyg 1998;59(4):513-8.

8. Marlar, T., K. Myat Phone and Yu S. Aye et al. Development of Resistance to Chloroquine by Plasmodium Vivax in Myanmar. Trans R Soc Trop Med Hyg 1995;89(3):3078.

9. White, N. Antimalarial Drug Resistance and Mortality in Falciparum Malaria [editorial]. Trop Med Int Health 1999;4(7):46970.

10. Hemme, F. and F. Gay. Internal Quality Control of the Malaria Microscopy Diagnosis for 10 Laboratories on the

\section{Table. Initial Malaria Assessment in Complex Humanitarian Emergencies}

Epidemiologic

Disease endemicity ${ }^{1}$

Prevalent Plasmodium species

First-line therapy

Antimalarial drug resistance

Immunity levels ${ }^{2}$

\section{Environmental}

Proximity to water

Proximity to agriculture

Proximity to forests

Temperature

Rainfall

\section{Entomologic}

Anopheline species

Biting behavior

Resting behavior

Vector breeding sites

Vectorial capacity ${ }^{3}$

1 Endemicity refers to the amount or severity of malaria in an area. Quantifying endemicity can be done by calculating spleen rates or parasite positivity rates (Hypoendemic: spleen rate/slide positivity in children (2-9 years) $<10 \%$; Mesoendemic: spleen rate/slide positivity in children (2-9 years) $11 \%-50 \%$; Hyperendemic: spleen rate/slide positivity in children ( $2-9$ years) $>50 \%$ and $>25 \%$ in adults; and Holoendemic: spleen rate/slide positivity in children (2-9 years) constantly $>75 \%$ but low adult spleen rates

2 Often relates to disease endemicity in country of origin.

${ }^{3}$ Vectorial capacity is term used to express the malaria transmission risk. It can be expressed as $C=\left(\mathrm{ma}^{2} \mathrm{p}^{\mathrm{n}} /-\log _{\mathrm{e}} \mathrm{p}\right)$ where ma is number of times a person is bitten per day, $\mathrm{p}^{\mathrm{n}}$ is proportion of vector population surviving incubation period or parasite. 
Thai-Myanmar Border. Southeast Asian J Trop Med Pub Health 1998;29(3):529-36.

11. Shiff, C. J., Z. Premji, and J. N. Minjas. The Rapid Manual ParaSight-F Test A New Diagnostic Tool for Plasmodium Falciparum Infection. Trans $R$ Soc Trop Med Hyg 1993;87(6):646-8.

12. Lackritz, E. M., C. C. Campbell, T. K Ruebush et al. Effect of Blood Transfusion on Survival Among Children in a Kenyan Hospital [see comments]. Lancet 1992;340(8818):524-8.

13. Zucker, J. R., B. A. Perkins, and H. Jafari et al. Clinical Signs for the Recognition of Children With Moderate or Severe Anaemia in Western Kenya. Bull World Health Organ 1997;75(Suppl 1):97-102.

14. Lewis, S. M., G. J. Stott, and K. J. Wynn. An Inexpensive and Reliable New Haemoglobin Colour Scale for Assessing Anaemia [see comments]. I Clin Pathol 1998;51(1):21-4.

15. Nosten, F., T. T.Hien, and N. J. White. Use of Artemisinin Derivatives for the Control of Malaria. Medecine Tropicale 1998;58(3 Suppl):45-9.

16. Warrell, D. A., M. E. Molyneux, and P. F. Beales .Severe and Complicated Malaria. Trans R Soc Trop Med Hyg 1990;84(Supplement 2).

17. Van Hensbroek, M. B., Jones S. Morris, S. Meisner et al. Iron, But Not Folic Acid, Combined With Effective Antimalarial Therapy Promotes Haematological Recovery in African Children After Acute Falciparum Malaria. Trans $R$ Soc Trop Med Hyg 1995;89(6):672-6.

18. Gilles, H. M., J. B. Lawson, and M. Sibelas et al. Malaria, Anaemia and Pregnancy. Ann Trop Med Parasitol 1969;63(2):245-63.

19.Steketee, R. W., J.J.Wirima, and L. Slutsker et. al.Malaria Treatment and Prevention in Pregnancy: Indications for Use and Adverse Events Associated with use of Chloroquine or Mefloquine. Am J Trop Med Hyg 1996;55(1):50-6.

20. Nosten, F., J. Karbwang, and N. J. White et al. Mefloquine Antimalarial Prophylaxis in Pregnancy: Dose Finding and PharmacokineticStudy. BrJClin Pharmacol 1990;30(1):79-85.

21. Parise, M. E, J.G. Ayisi, and B.L. Nahlen et al. Efficacy of Sulfadoxine-Pyrimethamine for Prevention of Placental Malaria in an Area of Kenya With a High Prevalence of Malaria and Human Immunodeficiency Virus Infection. Am J Trop Med Hyg 1998;59(5):813-22.

22. Shulman, C. E., E. K. Dorman, and F.Cutts et al. Intermittent Sulphadoxine-Pyrimethamine to Prevent Severe Anaemia Secondary to Malaria in Pregnancy: A
Randomised Placebo-Controlled Trial. Lancet 1999;353(9153):632-6.

23. Schultz, L. J., R.W. Steketee and A. Macheso et al. The Efficacy of Antimalarial Regimens Containing Sulfadoxine- Pyrimethamine and / or Chloroquine in Preventing Peripheral and Placental Plasmodium Falciparum Infection Among Pregnant Women in Malawi. Am J Trop Med Hyg 1994;51(5):515-22.

24. Najera, J. A., R. L. Kouznetzsov, and C. Delacollette. Malaria Epidemics: Detection and Control, Forcasting and Prevention: Geneva, WHO, 1998.

25. Centers for Disease Control and Prevention. Famine-Affected, Refugee, and Displaced Populations: Recommendations for Public Health Issues. MMWR 1992;41(RR-13).

26. Hewitt, S., M. Kamal, and N.Muhammad et al. An Entomological Investigation of the Likely Impact of Cattle Ownership on Malaria in an Afghan Refugee Camp in the North West Frontier Province of Pakistan. Med Vet Entomol 1994;8(2):160-4.

27. Bouma, M. and M. Rowland. Failure of Passive Zooprophylaxis: Cattle Ownership in Pakistan is Associated With a Higher Prevalence of Malaria [see comments]. Trans $R$ Soc Trop Med Hyg 1995;89(4):351-3.

28. Nosten. Personal Communication, October, 1999.
29. Glass, R. I., W. Cates Jr., and P. Nieburg et al. Rapid Assessment of Health Status and Preventive-medicine Needs of Newly Arrived Kampuchean Refugees, Sa Kaeo, Thailand. Lancet 1980;1(8173):868-72.

30. Toole, M. J. and R. J. Waldman. An Analysis of Mortality Trends Among Refugee Populations in Somalia, Sudan, and Thailand. Bull World Health Organ 1988;66(2):237-47.

31. Shears, P., A. M. Berry, and R. Murphy et al. Epidemiological Assessment of the Health and Nutrition of Ethiopian Refugees in Emergency Camps in Sudan, 1985. BrMed J (Clin Res Ed) 1987;295(6593):3148.

32. Rutherford, G. W. and A. E.Mahanjane. Morbidity and Mortality in the Mozambican Famine of 1983: Prevalence of Malnutrition and Causes and Rates of Death and Illness Among Dislocated Persons in Gaza and Inhambane Provinces. J Trop Pediatr 1985;31(3):143-9.

33. Chambers, R. Rural Appraisal: Rapid, Relaxed and Participatory: Brighton, Institute of Development Studies, 1992.

\section{Note}

The views expressed within this paper are solely those of the authors and do not reflect those of the United States Public Health Service.

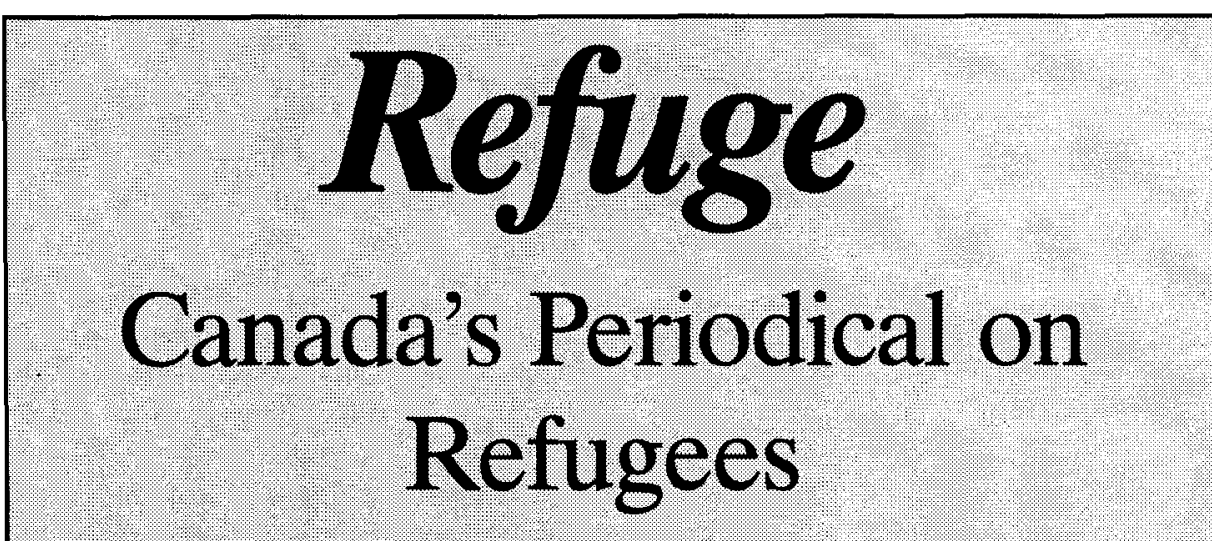

Published six times a year by the Centre for Refugee Studies, York University, Toronto.

Avallable from:

\section{Centre for Refugee Studies, York University \\ Suite 333, York Lanes, 4700 Keele St. \\ Toronto ON M3J 1P3}

Fax: (416) 736-5837 : Email: refuge@yorku.ca

http://www.yorku.ca/research/crs 